\title{
Determination of potential function in contact problems
}

\author{
F. Sharafbafi \& S. Adibnazari \\ Aerospace Engineering Department, Sharif University of Technology, \\ Tehran, Iran
}

\begin{abstract}
In this paper, a relation is introduced that simplifies the determination of the Muskhelishvili's potential function in plane contact problems. The relation is $\Phi(z)=1 / 2[p(z)-i q(z)]$, which is correct for all uncoupled-elastic contact problems. This relation is proved in a mathematical way and utilized to obtain the potential function in several contact problems. A complete agreement has been observed between our results and the potential functions that have been obtained from complicated methods in the past. Utilization of the relation simplifies the solution of contact problems and analytical calculation of the stress and displacement fields, which is helpful in the analytical studies of contact mechanics. It also may lead to the design of better cutting tools or fretting fatigue test pads.

Keywords: contact mechanics, uncoupled-elastic plane contacts, analytical solution, Muskhelishvili's potential function, a simple relation.
\end{abstract}

\section{Introduction}

A routine procedure for solving analytically plane contact problems is the following: find pressure and shear distributions from the contact fundamental equations and substitute those into the Muskhelishvili's integral equation to obtain the contact potential function. The contact stress and displacement fields are achievable through the determined potential function [1-3]. Since the Muskhelishvili's integral equation is a complex singular integral equation, calculation of the potential function, in this way, is not so simple. Therefore, the closed-form potential function of a few contact problems has been obtained. For a contact problem with complicated geometry, there are other procedures to 
obtain the potential function. The most common procedure is that of achieving the Chebyshev expansion of the pressure and shear distributions and putting them into the integral equation to obtain the expanded potential function. However, this method does not give the closed-form potential function because the Chebyshev series are not bounded [4]. The convergence of the Legendre series is Legendre expansion of the traction distributions instead of the Chebyshev expansion [5]. The other procedure to obtain the potential function is employing the Bertrand-Poincare's lemma to reverse the order of integration of the pressure and shear distributions and the Muskhelishvili's integral in order to simplify the integration procedure $[6,7]$.

Recently, a new method for finding the pressure distribution functions has been introduced. By arranging the flat punches along the contact profile and superposing the pressure distribution functions of the flat punches, for any contact profile, the pressure distribution function can be obtained in terms of a hyper-geometric function. The related potential function can be obtained through the superposing of the potential functions of flat punches along the contact profile $[8,9]$. Additionally, Finite Elements Modelling (FEM) has been utilized to solve contact problems and obtaining the potential function [10].

In this paper, it is proved that the potential function of an uncoupled contact problem can be obtained directly through the pressure and shear distributions by utilizing a simple relation such as $\Phi(z)=1 / 2[p(z)-i q(z)]$. This relation bypasses the Muskhelishvili's integral equation and simplifies the procedure of analytical solution of the contact problems. Since the relation is correct for all uncoupled contact problems such as complete, incomplete, multi-region and rough contacts, it is necessary to define a generalized contact problem before proving the relation. The generalized contact problem is defined in the "Theory" section of this paper. The relation is proved in the "Proof of the Relation" section of this paper. In the "Case Studies" section, several important contact problems are considered and for each of them, the potential function is obtained through the simple relation. The results were compared with the potential functions calculated by others. A complete agreement has been observed between them.

\section{Theory}

In this section, a generalized contact problem is considered. Although it might be repetitive, it is helpful to rewrite it in order to expose assumptions and equations essential for proving the simple relation.

Consider two elastic bodies $S_{1}$ and $S_{2}$, which are in contact with each other. According to figure 1, the body $S_{1}$ occupies the upper half-plane and the body $S_{2}$ occupies the lower half-plane. Along the boundaries of the bodies, there might be several contact segments namely $\sum_{i=1}^{n}\left[a_{i}, b_{i}\right]$. For the considered contact problem, the following usual assumptions have been made: 


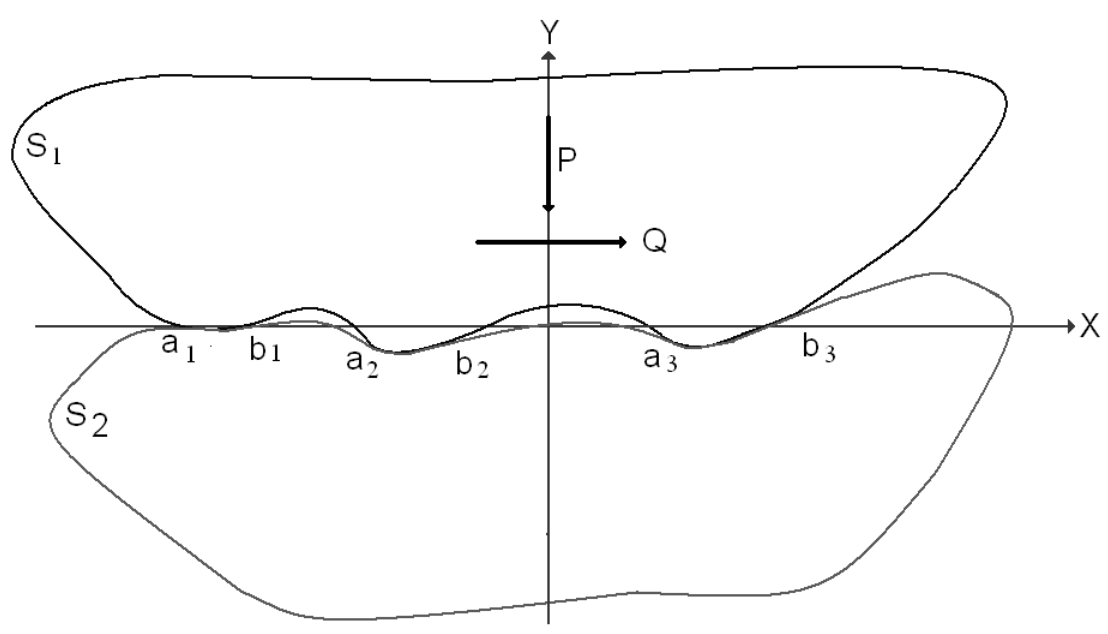

Figure 1: Contact of two bodies with several contact segments.

1- Each of the bodies can be approximated with a half-plane to achieve nonconformal contact problem.

2- Each contact length $L_{i}=\left[a_{i}, b_{i}\right]$ is very small in comparison with the dimensions of the bodies.

3- There is no rotation of contact bodies.

4- The stresses in $S_{1}$ and $S_{2}$ vanish at infinity.

5- The profiles of the boundaries are known before applying the external forces. The external normal and shear forces produce pressure and shear tractions on the contact surfaces. For the lower body $S_{2}$, the pressure distribution function $p(x)$ and the shear distribution function $q(x)$ are defined as

$$
p(x)=\sigma_{y y}(x, 0) \quad \& \quad q(x)=\tau_{x y}(x, 0)
$$

The positive direction of the pressure distribution function is the same as the positive $y$-axis [4]. It is assumed that, $p(x)$ and $q(x)$ satisfy the Hölder condition on the contact length and vanish at infinity [1]. The Muskhelishvili's potential function is defined as

$$
\Phi(z)=\frac{1}{2 \pi i} \int_{L} \frac{p(x)-i q(x)}{x-z} d x
$$

The potential function $\Phi(z)$ is a holomorphic complex function throughout the plane, except in the contact segments of the $x$-axis. Therefore, along the contact zones, Plemelj formulas can be written as

$$
\begin{gathered}
\Phi^{+}(x)-\Phi^{-}(x)=p(x)-i q(x) \\
\Phi^{+}(x)+\Phi^{-}(x)=\frac{1}{\pi i} \int_{L} \frac{p(\xi)-i q(\xi)}{\xi-x} d \xi
\end{gathered}
$$


It is well known that in plane contact problems, the fundamental equations are coupled singular integral equations, which relate the relative surface vertical overlap function $h(x)$ and the horizontal overlap function $g(x)$, to the contact pressure and shear distribution functions as follows:

$$
\begin{aligned}
& \frac{1}{A} \frac{\partial h(x)}{\partial x}=\frac{1}{\pi} \int_{L} \frac{p(\xi)}{(x-\xi)} d \xi-\beta q(x) . \\
& \frac{1}{A} \frac{\partial g(x)}{\partial x}=\frac{1}{\pi} \int_{L} \frac{q(\xi)}{(x-\xi)} d \xi+\beta p(x)
\end{aligned}
$$

where

$$
\begin{gathered}
A=\frac{\kappa_{1}+1}{4 \mu_{1}}+\frac{\kappa_{2}+1}{4 \mu_{2}} \\
\beta=\frac{\mu_{2}\left(\kappa_{1}-1\right)-\mu_{1}\left(\kappa_{2}-1\right)}{\mu_{2}\left(\kappa_{1}+1\right)+\mu_{1}\left(\kappa_{2}+1\right)}
\end{gathered}
$$

in which $\kappa=(3-v) /(1+v)$ is for plane stress and $\kappa=(3-4 v)$ is for plane strain conditions. The overlap functions $h(x)$ and $g(x)$ can be presented as follows:

$$
\begin{aligned}
& h(x)=v_{1}-v_{2} \\
& g(x)=u_{1}-u_{2}
\end{aligned}
$$

where $v_{1}$ and $v_{2}$ are the vertical and $u_{1}$ and $u_{2}$ are the horizontal components of the displacement of the contacting surfaces of the two bodies.

If Dundur's constant $\beta$ is zero ( $\beta=0)$, that is to say if the materials of the bodies are similar or the relation $\frac{\mu_{2}}{\mu_{1}}=\frac{k_{2}-1}{k_{1}-1}$ holds, the contact equations become decoupled.

Therefore, eqns (5), (6) reduce to

$$
\begin{aligned}
& \frac{1}{A} h^{\prime}(x)=\frac{1}{\pi} \int_{L} \frac{p(\xi) d \xi}{x-\xi} \\
& \frac{1}{A} g^{\prime}(x)=\frac{1}{\pi} \int_{L} \frac{q(\xi) d \xi}{x-\xi}
\end{aligned}
$$

Equations (11) and (12) are the fundamental equations in the uncoupled contact problems.

\section{Proof of the relation}

For a generalized and uncoupled contact problem, another boundary condition is obtained through the fundamental contact equations. Multiplying eqn (11) by $i$ and adding it to the eqn (12), yields 


$$
\frac{1}{A}\left[g^{\prime}(x)+i h^{\prime}(x)\right]=\frac{1}{\pi i} \int_{L} \frac{p(\xi)-i q(\xi)}{\xi-x} d \xi
$$

which is a Cauchy singular integral equation of the first kind. The solution (also known as inversion) of eqn (13) is

$$
p(x)-i q(x)=\frac{\omega(x)}{\pi A} \int_{L}^{h^{\prime}(\xi)-i g^{\prime}(\xi)} \frac{\omega(\xi)(\xi-x)}{\omega \xi}+\Omega(x) \omega(x)
$$

where $\omega(x)$ is the weight function and $\Omega(x)$ is a polynomial of up to an order less than $n$, where $n$ is the number of the contact zones. In accordance with the contact geometry and the behavior of surface tractions at the ends of each contact zone, $\omega(x)$ and $\Omega(x)$ are then determined [1,2]. The results are summarized in table 1 for multiple contact zones.

Table 1: Weight function $\omega(x)$ and the polynomial function $\Omega(x)$ for different contact boundary conditions

\begin{tabular}{|l|l|l|}
\hline $\begin{array}{l}\text { Boundary } \\
\text { conditions }\end{array}$ & Weight function $\omega(x)$ & $\Omega(x)$ \\
\hline $\begin{array}{l}\text { Singular at all } \\
\text { end points }\end{array}$ & $\omega(x)=\left(\prod_{i=1}^{n}\left(x-a_{i}\right)\left(b_{i}-x\right)\right)^{-1 / 2}$ & $\Omega(x)=\sum_{i=0}^{n-1} c_{i} x^{i}$ \\
\hline $\begin{array}{l}\text { Non-singular at } \\
x=a_{j}\end{array}$ & $\omega(x)=\left(\prod_{j=1}^{m}\left(x-a_{j}\right)\left(\prod_{i=1}^{n}\left(x-a_{i}\right)\left(b_{i}-x\right)\right)^{-1 / 2}\right.$ & $\Omega(x)=\sum_{i=0}^{n-1} c_{i} x^{i}$ \\
\hline $\begin{array}{l}\text { Non-singular at } \\
x=b_{j}\end{array}$ & $\omega(x)=\left(\prod_{j=1}^{m}\left(b_{j}-x\right)\right)\left(\prod_{i=1}^{n}\left(x-a_{i}\right)\left(b_{i}-x\right)\right)^{-1 / 2}$ & $\Omega(x)=\sum_{i=0}^{n-1} c_{i} x^{i}$ \\
\hline $\begin{array}{l}\text { Non-singular at } \\
\text { all end points }\end{array}$ & $\omega(x)=\left(\prod_{i=1}^{n}\left(x-a_{i}\right)\left(b_{i}-x\right)\right)^{1 / 2}$ where & $\Omega(x)=\sum_{i=0}^{n-1} c_{i} x^{i}$ \\
\hline
\end{tabular}

On the other hand, comparing equations (4) and (13) results in:

$$
\Phi^{+}(x)+\Phi^{-}(x)=\frac{1}{A}\left[g^{\prime}(x)+i h^{\prime}(x)\right]
$$

This equation is a non-homogeneous Hilbert-Reimann linear problem and its solution is:

$$
\Phi(z)=\frac{\omega^{*}(z)}{2 \pi A} \int_{L} \frac{h^{\prime}(\xi)-i g^{\prime}(\xi)}{\omega^{*}(\xi)(\xi-z)} d \xi+\frac{\Omega^{*}(z) \omega^{*}(z)}{2}
$$

where $\omega^{*}(z)$ is a weight function and $\Omega^{*}(z)$ is a polynomial of up to an order less than $n$, where $n$ is the number of the contact zones. Since both of the 
weight functions $\omega(x)$ and $\omega^{*}(z)$ are dependent on the contact geometry and the behavior of surface tractions at the ends of each contact zone, it can be shown that $\omega^{*}(x)=\omega(x)$. Additionally, the order of both polynomials $\Omega(x)$ and $\Omega^{*}(z)$ are the same. Therefore it is possible to consider $\Omega^{*}(z)$ such that the relation $\Omega^{*}(x)=\Omega(x)$ holds. Comparison of eqn (14) and (16) yields to:

$$
\Phi(z)=1 / 2[p(z)-i q(z)]
$$

In other words, if the closed form of the pressure and shear distribution functions, $p(x)$ and $q(x)$, are known, for determination of the potential function of the contact problem, it is enough to replace $z$ for $x$ in the pressure and shear distribution functions, $p(z)$ and $q(z)$, and utilize eqn (17) to obtain the potential function. Due to the fact that for all contact profiles, $\omega^{*}(x)=\omega(x)$ and $\Omega^{*}(x)=\Omega(x)$, eqn (17) holds true for all contact profiles: be it Hertzian, non-Hertzian, with or without singularities or even rough contacts. It is noteworthy that the imaginary part of the pressure or shear distribution functions should not be omitted since, although the imaginary parts have no meaning in the pressure and shear distributions, they are required for computing the potential function through the eqn (17). Additionally, if the traction distribution functions are defined in the normalized plane, the potential function obtained through eqn (17) is consequently in the normalized complex plane.

By comparing eqns (2) and (17), we can rewrite the Muskhelishvili's integral relation as:

$$
\Phi(z)=\frac{1}{2 \pi i} \int_{L} \frac{p(\xi)-i q(\xi)}{\xi-z} d \xi=\frac{p(z)-i q(z)}{2}
$$

Of course, this relation is correct for uncoupled-elastic contact problems.

Determining the potential function in closed form makes it possible to obtain the closed form relations for the displacement and stress components through the following equations:

$$
\begin{gathered}
\sigma_{x x}+\sigma_{y y}=4(\operatorname{Re}(\Phi(z)) \\
\sigma_{y y}-\sigma_{x x}+2 i \sigma_{x y}=2\left[(\bar{z}-z) \Phi^{\prime}(z)-\bar{\Phi}(z)-\Phi(z)\right] \\
2 \mu\left(\frac{\partial u}{\partial x}+i \frac{\partial v}{\partial y}\right)=(\bar{z}-z) \overline{\Phi^{\prime}}(\bar{z})+\Phi(\bar{z})+\kappa \Phi(z)
\end{gathered}
$$

\section{Case studies}

Although the simple relation, eqn (17), is proved in mathematics, it is important to find the conformity of the potential function obtained through the relation and those introduced in the literature. It is noteworthy that the closed form of the potential function has been achieved only for a few simple contact problems. 
In this section, four important contact problems, which had analytical solutions, are considered such as 1- the classic Hertz contact problem, 2- contact of a flat punch with half plane, 3- contact problem of a symmetrical wedge with half plane and 4- contact of a punch generating uniform pressure. Figure 2 shows profile of the indenters and pressure distribution of each contact problem. For each mentioned contact problem, the potential function has been extracted from eqn (17) and compared with the existing results in the literature. Agreement between these two potential functions, confirm the accuracy of the simple relation.

The asterisk sign on the parameters shows that the related equation had been achieved before.
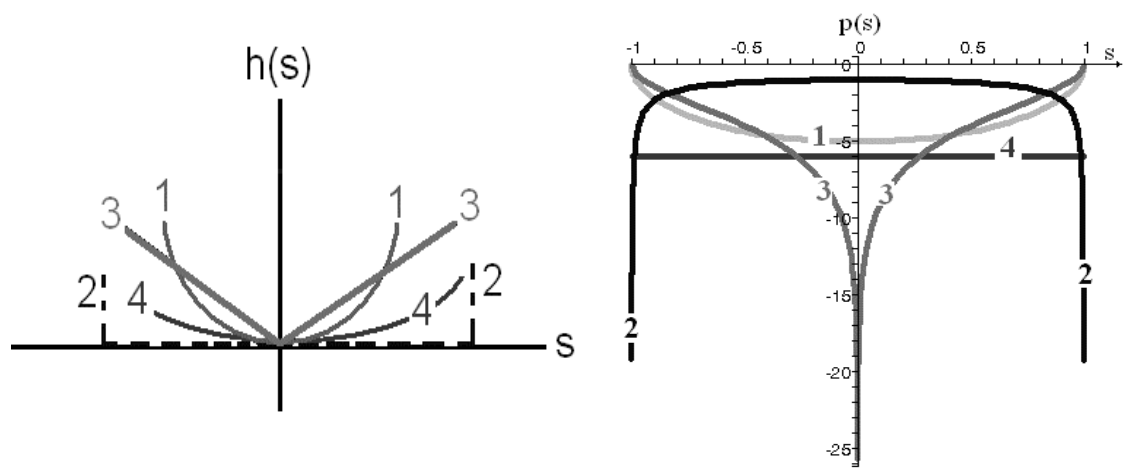

Figure 2: Indenters profiles (left) and pressure distributions (right) of the considered contact problems.

\subsection{Hertz contact problem}

In 1882, Hertz introduced and solved contact problem of two cylinders. The pressure distribution of the famous Hertz contact problem is:

$$
p^{*}(s)=\frac{-k a}{A} \sqrt{1-s^{2}} \quad s \in[-1,1]
$$

where $a$ is half of contact length and $s$ is the normalized axis along the contact surface. The potential function for this contact problem was calculated by integrating the Cauchy integral $[4$, table 3.5$]$ :

$$
\Phi^{*}(z)=\int_{-1}^{1} \frac{p(\xi)}{\xi-z} d \xi=\left(-\frac{k a i}{2 A}\right)\left[z-\sqrt{z^{2}-1}\right]
$$

It is seen that substitution of the achieved pressure distribution eqn (22) into the eqn (17) does not yield the same result as eqn (23) and is a major nonconformity. However, this discrepancy must have occurred because of the imaginary part of the pressure distribution. To obtain the imaginary part of the pressure distribution, the contact problem is solved again and the complete form of the pressure distribution is obtained as follows 


$$
p(s)=-\left(\frac{k a}{A}\right)\left[\sqrt{1-s^{2}}+i s\right]
$$

It is obvious that the real part of the pressure distribution function eqn (24) is the same as the pressure distribution function in eqn (22). Substituting the complete pressure distribution function eqn (24) into the eqn (17) gives:

$$
\Phi(z)=-\left(\frac{k a}{2 A}\right)\left[\sqrt{1-z^{2}}+i z\right]=\left(-\frac{k a i}{2 A}\right)\left[z-\sqrt{z^{2}-1}\right]
$$

The potential function achieved through the eqn (17) is exactly the same as the previously obtained potential function eqn (23). The obtained potential function is plotted as a two dimensional function in figure 3-1.

\subsection{Contact of a flat punch with a half plane}

As a second example, a flat punch in contact with a half plane with no shear force or friction is considered. Muskhelishvili [1] has extracted pressure distribution and potential function as follows:

$$
\begin{gathered}
p^{*}(s)=\frac{-P}{a \pi \sqrt{1-s^{2}}} \\
\Phi^{*}(z)=\frac{-P}{2 a \pi \sqrt{1-z^{2}}}
\end{gathered}
$$

It is obvious that substitution of the pressure distribution function eqn (26) into the eqn (17) yields the same potential function as eqn (27). The obtained potential function is plotted as a two dimensional function in figure 3-2.

\subsection{Contact of a symmetrical wedge with a half plane}

Complete form of the pressure distribution of contact problem of a symmetrical wedge with a half plane can be obtained as:

$$
p(s)=\frac{-2 \phi}{\pi A}\left(\cosh ^{-1}\left(\frac{1}{|s|}\right)+\frac{\pi i}{2}\right)
$$

Substitution of the pressure distribution in eqn (17), gives the potential function:

$$
\Phi(z)=\frac{-p_{0}}{2}\left[\cosh ^{-1}\left(\frac{1}{|z|}\right)+\frac{\pi i}{2}\right]
$$

Simplifying the above equation leads to:

$$
\Phi^{*}(z)=\frac{-i p_{0}}{2} \sin ^{-1}\left(\frac{1}{z}\right)
$$

The latter form of the potential function is the same as the closed potential function obtained by Truman and Sackfield [11] for this contact problem. The obtained potential function is plotted as a two dimensional function in figure 3-3. 


\subsection{Contact of a punch generating uniform pressure}

Hills et al [4] obtained the profile of a punch which generates uniform pressure over the contacting half plane. Due to the specific profile of the punch, the pressure distribution is uniform and equal to:

$$
p(s)=-p_{0}=\frac{P}{2 a} \quad-1 \leq s \leq 1
$$

Substituting the pressure distribution in the Muskhelishvili's integral equation gives the potential function as:

$$
\Phi(z)=\frac{-p_{0}}{2 \pi i} \int_{-1}^{1} \frac{d s}{s-z}=\frac{-p_{0}}{2 \pi i} \ln \left(\frac{1-z}{-1-z}\right)=\frac{p_{0}}{2}+i \frac{p_{0}}{2 \pi} \ln \left(\frac{1-z}{1+z}\right)
$$
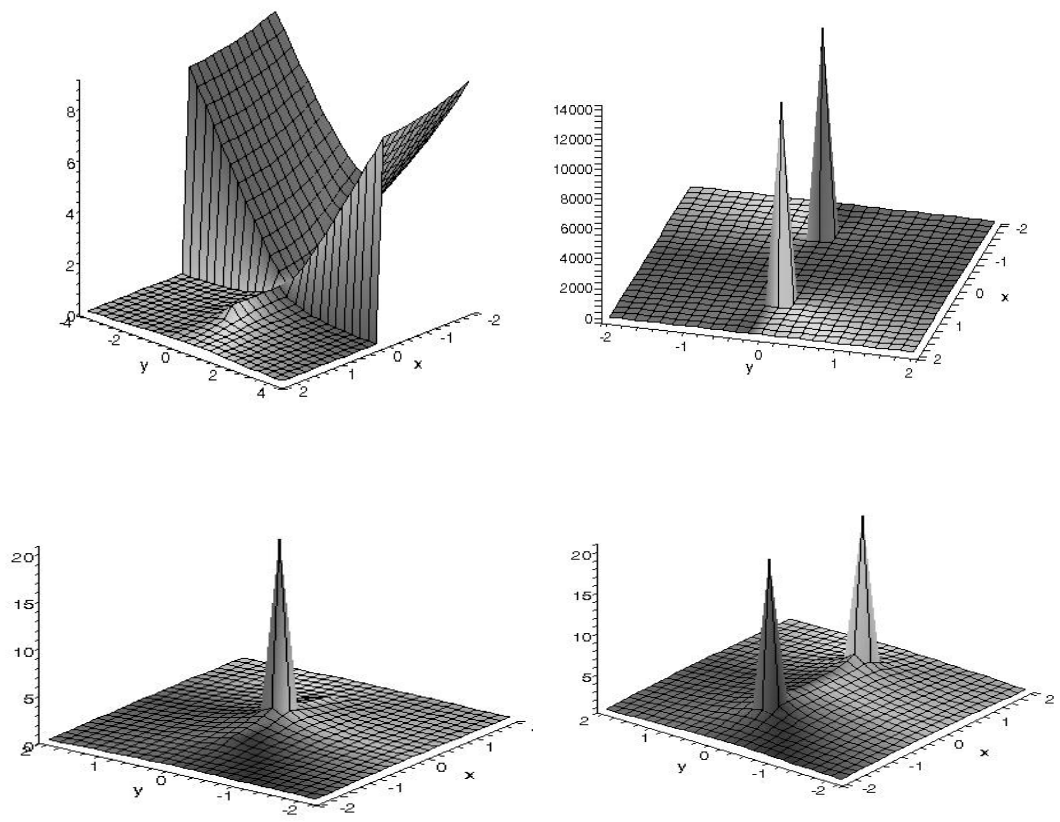

Figure 3: The potential functions of the related contact problems 3-1 Hertz contact (top- left), 3-2 Flat punch (top-right), 3-3 symmetrical wedge (bottom-left) \& 3-4 Punch generating uniform pressure (bottom- right).

This form of the potential helps to determine the imaginary part of the complete pressure distribution function. According the eqn (18), the complete form of the pressure distribution function will be equal to:

$$
p(z)=2\left\{\frac{p_{0}}{2}+i \frac{p_{0}}{2 \pi} \ln \left(\frac{1-z}{1+z}\right)\right\}=p_{0}+i \frac{p_{0}}{\pi} \ln \left(\frac{1-z}{1+z}\right)
$$


The real part of $p(z)$ is the same as the defined pressure distribution in eqn (31) and this is another confirmation of the eqn (17). The imaginary part of the uniform distribution is obtained as $\frac{p_{0}}{\pi} \ln \left(\frac{1-s}{1+s}\right)$. The obtained potential function is plotted as a two dimensional function in figure 3-4. The singularity of the potential function shown in the figure is very interesting.

\section{Conclusion}

Conformity of the potential function obtained through the simple relation with those obtained by complicated methods in the literature, shows the accuracy and simplicity of the relation. Therefore, in order to determine the potential function of an uncoupled contact problem, it is recommended to find the complete form of the pressure and shear distribution functions and utilize the simple relation $\Phi(z)=1 / 2[p(z)-i q(z)]$ to achieve the potential function.

\section{References}

[1] Muskhelishvili, N.I., Some Basic Problems of the Mathematical Theory of Elasticity, translated by Radok, JRM, Noordhoff Ltd, Groningen, Netherlands, 1963.

[2] Gladwell, G.M.L., Contact Problems in the Classical Theory of Elasticity, Sijthoff \& Noordhoff, Rockville, MD, 1980.

[3] Johnson, K.L., Contact Mechanics, Cambridge University Press, UK, 1987.

[4] Hills, D.A., Nowell, D. \& Sackfield, A. Mechanics of Elastic Contacts, Oxford Press, Butterworth-Heinemann, 1993.

[5] Badr, A.A., On the Numerical Treatment of the Contact Problem, Int. J. of Mathematics \& Materials Science, 23, No. 12, pp. 865-871, 2000.

[6] Sackfield, A., Dini, D. \& Hills, D.A., The Finite and Semi-infinite Tilted Flat but Rounded Punch, Int. J. of Solids and Structures, 42, pp. 49885009, 2005.

[7] Sackfield, A., Dini, D. \& Hills, D.A., The Tilted Shallow Wedge Problem, European Journal of Mechanics A/ Solids, 24, pp. 919-928, 2005.

[8] Jäger, J., A New Principle in Contact Mechanics, Journal of Tribology, 120, pp. 677- 683, 1998.

[9] Jäger, J., New Analytical and Numerical Results for Two-Dimensional Contact Profiles, International Journal of Solids and Structures, 39, pp. 959-972, 2002.

[10] Mackerle, J., Contact Mechanics: Finite Element and Boundary Elements Approach, a Bibliography (1995-1997) Finite Element Analysis, Dec 29, pp. 275-285, 1998.

[11] Truman, C.E. \& Sackfield, A., Closed-Form Solution for the Stress Fields Induced by Blunt Wedge Shaped Indenters in Elastic Half Planes, Int. J. Applied Mech., 68, pp. 817-819, 2001. 